Supplementary material for: Data-driven distinction between convective, frontal and mixed extreme rainfall events in radar data

S1. Sampled extreme events

The 39 sampled extreme events for 15-min, 1-hour and 24-hour durations can be seen in Table A, Table B and Table C respectively. 
Table A: Sampled 15-min extreme events with respect to the 17 variables selected for analysis

\begin{tabular}{|c|c|c|c|c|c|c|c|c|c|c|c|c|c|c|c|c|c|c|c|}
\hline $\begin{array}{l}15- \\
\min \end{array}$ & start & end & $\begin{array}{l}\text { duration } \\
{[\mathrm{h}]}\end{array}$ & $\begin{array}{l}\max \\
15 \mathrm{~min} \\
{[\mathrm{~mm} / \mathrm{hr}]}\end{array}$ & $\begin{array}{l}\text { ratio } \\
15 \text { min } \\
{[-]}\end{array}$ & $\begin{array}{l}\max \\
1 \mathrm{hr} \\
{[\mathrm{mm} / \mathrm{hr}]}\end{array}$ & $\begin{array}{l}\max \\
24 \mathrm{hr} \\
{[\mathrm{mm} / \mathrm{hr}]}\end{array}$ & $\begin{array}{l}\text { mean } \\
\text { wet A } \\
{[-]}\end{array}$ & $\begin{array}{l}\min \\
\text { depth } \\
{[\mathrm{mm}]}\end{array}$ & $\begin{array}{l}\text { max } \\
\text { depth } \\
{[\mathrm{mm}]}\end{array}$ & $\begin{array}{l}\text { mean } \\
\text { depth } \\
{[\mathrm{mm}]}\end{array}$ & $\begin{array}{l}\text { ratio } \\
\text { depth } \\
{[-]}\end{array}$ & $\begin{array}{l}\text { cell } \\
\text { num } \\
{[-]}\end{array}$ & $\begin{array}{l}\text { cell life } \\
\text { mean } \\
{[\mathrm{h}]}\end{array}$ & $\begin{array}{l}\text { cell life } \\
\text { max } \\
\text { [h] }\end{array}$ & $\begin{array}{l}\text { mean } \\
\text { vel } \\
{[\mathrm{m} / \mathbf{s}]}\end{array}$ & $\begin{array}{l}\text { sd } \\
\text { vel } \\
{[-]}\end{array}$ & $\begin{array}{l}\text { mean } \\
\text { dir } \\
\text { [deg.] }\end{array}$ & $\begin{array}{l}\text { sd } \\
\text { dir } \\
{[-]}\end{array}$ \\
\hline 1 & 14-05-2001 11:12 & 19-05-2001 01:02 & 109.83 & 56.30 & 35.00 & 18.20 & 1.05 & 0.26 & 17.91 & 50.58 & 29.36 & 1.72 & 9.00 & 0.36 & 1.08 & 16.64 & 5.55 & 47.98 & 0.42 \\
\hline 2 & 06-06-2001 00:57 & 09-06-2001 00:07 & 71.17 & 55.93 & 84.26 & 14.27 & 0.66 & 0.31 & 4.12 & 23.90 & 11.34 & 2.11 & 2.00 & 0.08 & 0.17 & 10.27 & 0.38 & 80.78 & 0.16 \\
\hline 3 & 30-06-2001 05:27 & $30-06-200121: 27$ & 16.00 & 87.33 & 22.92 & 28.73 & 1.91 & 0.41 & 5.41 & 45.83 & 18.11 & 2.53 & 7.00 & 0.43 & 0.83 & 12.09 & 9.77 & 87.23 & 0.58 \\
\hline 4 & $17-07-200105: 32$ & $21-07-2001 \quad 16: 52$ & 107.33 & 135.80 & 92.39 & 41.88 & 2.63 & 0.29 & 16.53 & 77.87 & 34.82 & 2.24 & 3.00 & 0.42 & 0.50 & 9.47 & 1.53 & 53.72 & 0.23 \\
\hline 5 & $02-08-2001 \quad 15: 27$ & $10-08-200122: 47$ & 199.33 & 63.30 & 44.03 & 18.95 & 1.24 & 0.27 & 27.90 & 86.38 & 55.80 & 1.55 & 13.00 & 0.25 & 1.00 & 14.29 & 4.27 & 85.08 & 0.61 \\
\hline 6 & $17-03-2002 \quad 12: 02$ & $22-03-200216: 07$ & 124.08 & 44.40 & 35.88 & 14.56 & 1.44 & 0.46 & 28.96 & 83.62 & 56.44 & 1.48 & 6.00 & 0.36 & 0.92 & 13.94 & 5.66 & 89.94 & 0.39 \\
\hline 7 & $19-08-2002 \quad 05: 32$ & $22-08-2002 \quad 04: 37$ & 71.08 & 113.97 & 43.20 & 37.53 & 3.95 & 0.37 & 27.28 & 102.48 & 53.13 & 1.93 & 8.00 & 0.44 & 1.00 & 9.37 & 3.77 & 16.81 & 0.68 \\
\hline 8 & 05-10-2002 06:12 & 08-10-2002 00:07 & 65.92 & 49.43 & 31.12 & 25.62 & 2.77 & 0.39 & 10.59 & 69.32 & 36.20 & 1.92 & 9.00 & 0.47 & 1.83 & 13.83 & 10.35 & 143.45 & 0.76 \\
\hline 9 & 08-06-2003 10:32 & 08-06-2003 21:02 & 10.50 & 119.73 & 31.86 & 40.78 & 2.13 & 0.49 & 3.63 & 51.17 & 17.48 & 2.93 & 8.00 & 0.19 & 0.42 & 12.92 & 4.55 & 28.54 & 0.53 \\
\hline 10 & $17-06-2003 \quad 17: 17$ & $20-06-200300: 02$ & 54.75 & 59.17 & 37.69 & 21.68 & 1.32 & 0.22 & 3.08 & 32.84 & 12.24 & 2.68 & 7.00 & 0.18 & 0.33 & 8.65 & 3.08 & 106.13 & 0.78 \\
\hline 11 & $30-06-2003$ 07:57 & $05-07-2003 \quad 17: 37$ & 129.67 & 81.73 & 68.56 & 21.88 & 1.52 & 0.27 & 15.39 & 70.14 & 35.70 & 1.96 & 14.00 & 0.15 & 0.42 & 14.21 & 5.35 & 53.77 & 0.27 \\
\hline 12 & $30-07-200305: 07$ & $31-07-2003$ 07:07 & 26.00 & 80.73 & 70.45 & 25.98 & 1.23 & 0.36 & 0.92 & 29.63 & 7.03 & 4.22 & 5.00 & 0.37 & 1.25 & 7.16 & 5.33 & 83.16 & 0.72 \\
\hline 13 & $10-09-200423: 47$ & $404: 17$ & 28.50 & 61.60 & 16.10 & 22.43 & 2.33 & 0.30 & 10.83 & 55.83 & 26.89 & 2.08 & 17.00 & 0.45 & 1.08 & 14.02 & 4.22 & 67.10 & 0.55 \\
\hline 14 & 29-04-2005 01:32 & $513: 47$ & 300.25 & 59.27 & 50.94 & 18.26 & 1.37 & 0.32 & 47.22 & 116.53 & 68.59 & 1.70 & 8.00 & 0.17 & 0.50 & 13.06 & 4.96 & 63.02 & 0.33 \\
\hline 15 & 03-06-2005 15:12 & 06-06-2005 11:42 & 68.50 & 45.80 & 33.98 & 14.14 & 1.03 & 0.29 & 7.18 & 40.39 & 22.10 & 1.83 & 10.00 & 0.25 & 0.58 & 13.28 & 5.21 & 87.85 & 0.50 \\
\hline 16 & 29-06-2005 00:27 & 03-07-2005 00:27 & 96.00 & 92.33 & 33.47 & 41.19 & 4.43 & 0.36 & 37.68 & 138.45 & 78.02 & 1.77 & 11.00 & 0.36 & 1.17 & 6.46 & 4.91 & 38.70 & 1.58 \\
\hline 17 & $18-07-200522: 42$ & $521: 52$ & 311.17 & 130.77 & 98.47 & 7 & 2 & 0.28 & 44.18 & 124.88 & 70.28 & 1.78 & 10.00 & 0.28 & 0.83 & 13.39 & 7.61 & 62.03 & 0.52 \\
\hline 18 & 29-05-2006 06:12 & 06 17:02 & 82.83 & 41.67 & 49.19 & 16.53 & 0.92 & 0.28 & 8.33 & 30.73 & 16.57 & 1.85 & 5.00 & 0.35 & 1.00 & 14.88 & 7.95 & 92.72 & 0.75 \\
\hline 19 & 24-08-2006 08:17 & $31-08-200622: 47$ & 182.50 & 56.20 & 35.49 & 22.01 & 1.49 & 0.25 & 23.08 & 80.91 & 51.42 & 1.57 & 27.00 & 0.29 & 0.92 & 11.74 & 5.73 & 103.27 & 0.74 \\
\hline 20 & 24-05-2007 22:07 & 29-05-2007 19:02 & 116.92 & 88.50 & 85.05 & 25.80 & 1.58 & 0.51 & 26.97 & 60.99 & 39.05 & 1.56 & 4.00 & 0.27 & 0.75 & 15.22 & 4.51 & 32.53 & 0.58 \\
\hline 21 & 06-08-2007 14:17 & $720: 02$ & 101.75 & 182.43 & 100.01 & 3 & 4 & 0.59 & 46.83 & 184.46 & 80.74 & 2.28 & 1.00 & 1.50 & 1.50 & 7.05 & 2.34 & 46.28 & 0.32 \\
\hline 22 & $14-05-2008$ 12:02 & $801: 47$ & 85.75 & 135.27 & 127.91 & 67.80 & 3.4 & 0.29 & 5.93 & 91.52 & 19.12 & 4.79 & 4.00 & 0.31 & 0.58 & 6.95 & 5.48 & 77.29 & 0.83 \\
\hline 23 & 05-07-2009 12:02 & $13-07-200900: 17$ & 180.25 & 75.40 & 56.03 & 21.61 & 1.70 & 0.28 & 32.76 & 92.73 & 56.21 & 1.65 & 9.00 & 0.20 & 0.33 & 9.91 & 4.33 & 74.13 & 0.32 \\
\hline 24 & $16-07-2009$ 15:22 & $25-07-2009$ 18:12 & 218.83 & 94.23 & 84.16 & 31.49 & 1.66 & 0.26 & 36.08 & 73.13 & 52.89 & 1.38 & 7.00 & 0.29 & 0.67 & 14.51 & 6.71 & 76.76 & 0.49 \\
\hline 25 & $10-07-201023: 02$ & $15-07-201008: 07$ & 105.08 & 71.33 & 36.85 & 21.58 & 0.98 & 0.19 & 14.41 & 36.35 & 22.23 & 1.64 & 9.00 & 0.31 & 0.75 & 15.86 & 4.99 & 41.19 & 0.53 \\
\hline 26 & $10-08-2$ & $12-08$ & 36.83 & 72.57 & 49 & 9 & 2 & 0 & 10.74 & 59.63 & 20.81 & 2.87 & 6.00 & 49 & 1.83 & 9.15 & 7.06 & 44.24 & 0.75 \\
\hline 27 & $25-08-2010 \quad 15: 32$ & $02: 27$ & 154.92 & 60.27 & 42. & 2 & 2 & 0.4 & 47.47 & 120.27 & 78.13 & 1.54 & 16.00 & 0.31 & 1.67 & 12.34 & 5.72 & 112.53 & 0.63 \\
\hline 28 & 04-06-2011 18:42 & 08-06-2011 11:12 & 88.50 & 96.70 & 44.82 & 41.74 & 2.41 & 0.32 & 13.66 & 89.42 & 41.10 & 2.18 & 15.00 & 0.27 & 1.08 & 7.83 & 4.59 & 25.72 & 1.05 \\
\hline 29 & $15-06-201105: 32$ & 03-07-2011 14:47 & 441.25 & 55.73 & 67.18 & 19.92 & 1.47 & 0.28 & 40.16 & 99.09 & 70.21 & 1.41 & 16.00 & 0.22 & 0.42 & 13.54 & 4.85 & 65.98 & 0.53 \\
\hline 30 & $16-07-2011 \quad 12: 22$ & 22-07-2011 21:37 & 153.25 & 86.30 & 74.44 & 27.15 & 1.41 & 0.32 & 25.96 & 69.60 & 39.76 & 1.75 & 6.00 & 0.18 & 0.50 & 9.43 & 3.95 & 77.12 & 1.12 \\
\hline 31 & 11-08-2011 03:02 & $16-08-2$ & 13075 & 5507 & 36.90 & 1747 & 14 & 0.29 & 20.90 & 53.62 & 38.00 & 1.41 & 5.00 & 0.35 & 0.67 & 12.31 & 4.86 & 69.10 & 0.67 \\
\hline 32 & $10-07-2012 \quad 10: 12$ & 21-07-2012 21:42 & 275.50 & 108.87 & 87.76 & 31.33 & 1.75 & 0.36 & 53.72 & 145.63 & 101.10 & 1.44 & 6.00 & 0.31 & 1.33 & 10.24 & 4.08 & 71.75 & 0.53 \\
\hline 33 & $23-08-2012$ 20:22 & $26-08-2012$ 22:12 & 73.83 & 53.77 & 37.89 & 19.19 & 1.75 & 0.40 & 14.79 & 56.55 & 29.16 & 1.94 & 11.00 & 0.20 & 0.58 & 13.91 & 6.79 & 99.17 & 0.67 \\
\hline 34 & 06-04-2013 05:02 & 14-04-2013 04:12 & 191.17 & 34.40 & 73.51 & 9.13 & 0.66 & 0.38 & 14.08 & 33.75 & 23.64 & 1.43 & 1.00 & 1.08 & 1.08 & 12.61 & 1.88 & 81.87 & 0.34 \\
\hline 35 & 07-05-2013 09:12 & $01312: 52$ & & 67.93 & 62.69 & 32.58 & 1.50 & 0.29 & 0.88 & 39.08 & 9.22 & 4.24 & 4.00 & 0.60 & 1.00 & 7.75 & 4.38 & 295.46 & 0.85 \\
\hline 36 & 19-06-2013 18:02 & $25-06-2013 \quad 15: 12$ & 141.17 & 364.70 & 202.71 & 110.81 & 5.40 & 0.29 & 16.73 & 155.09 & 58.70 & 2.64 & 3.00 & 0.53 & 0.67 & 11.25 & 4.04 & 357.37 & 0.41 \\
\hline 37 & $22-07-2013$ 11:42 & $31-07-2013$ 07:02 & 211.33 & 121.23 & 95.53 & 59.63 & 3.16 & 0.22 & 13.41 & 125.56 & 39.62 & 3.17 & 14.00 & 0.36 & 0.92 & 7.30 & 6.85 & 119.31 & 1.61 \\
\hline 38 & $24-08-2013 \quad 13: 32$ & $26-08-2013 \quad 15: 27$ & 49.92 & 56.53 & 56.00 & 19.95 & 0.93 & 0.26 & 2.33 & 24.46 & 9.75 & 2.51 & 3.00 & 0.36 & 0.92 & 9.32 & 3.38 & 245.25 & 0.61 \\
\hline 39 & 06-09-2013 13:57 & $14-09-2013$ 18:57 & 197.00 & 93.87 & 55.13 & 34.85 & 2.18 & 0.37 & 61.10 & 110.71 & 86.76 & 1.28 & 11.00 & 0.24 & 0.58 & 12.96 & 6.09 & 340.25 & 1.80 \\
\hline
\end{tabular}


Table B: Sampled 1-hour extreme events

\begin{tabular}{|c|c|c|c|c|c|c|c|c|c|c|c|c|c|c|c|c|c|c|c|}
\hline $\begin{array}{l}1- \\
\text { hour }\end{array}$ & start & end & $\begin{array}{l}\text { duration } \\
{[\mathrm{h}]}\end{array}$ & $\begin{array}{l}\max \\
15 \mathrm{~min} \\
{[\mathrm{~mm} / \mathrm{hr}]}\end{array}$ & $\begin{array}{l}\text { ratio } \\
15 \text { min } \\
{[-]}\end{array}$ & $\begin{array}{l}\max \\
1 \mathrm{hr} \\
{[\mathrm{mm} / \mathrm{hr}]}\end{array}$ & $\begin{array}{l}\max \\
24 \mathrm{hr} \\
{[\mathrm{mm} / \mathrm{hr}]}\end{array}$ & $\begin{array}{l}\text { mean } \\
\text { wet A } \\
{[-]}\end{array}$ & $\begin{array}{l}\text { min } \\
\text { depth } \\
{[\mathrm{mm}]}\end{array}$ & $\begin{array}{l}\max \\
\text { depth } \\
{[\mathrm{mm}]}\end{array}$ & $\begin{array}{l}\text { mean } \\
\text { depth } \\
{[\mathrm{mm}]}\end{array}$ & $\begin{array}{l}\text { ratio } \\
\text { depth } \\
{[-]}\end{array}$ & $\begin{array}{l}\text { cell } \\
\text { num } \\
{[-]}\end{array}$ & $\begin{array}{l}\text { cell life } \\
\text { mean } \\
{[\mathrm{h}]}\end{array}$ & $\begin{array}{l}\text { cell life } \\
\max \\
{[\mathrm{h}]}\end{array}$ & $\begin{array}{l}\text { mean } \\
\text { vel } \\
{[\mathrm{m} / \mathrm{s}]}\end{array}$ & $\begin{array}{l}\text { sd } \\
\text { vel } \\
{[-]}\end{array}$ & $\begin{array}{l}\text { mean } \\
\text { dir } \\
\text { [deg.] }\end{array}$ & $\begin{array}{l}\text { sd } \\
\text { dir } \\
{[-]}\end{array}$ \\
\hline 1 & 06-06-2001 00:57 & 09-06-2001 00:07 & 71.17 & 55.93 & 84.26 & 14.27 & 0.66 & 0.31 & 4.12 & 23.90 & 11.34 & 2.11 & 2.00 & 0.08 & 0.17 & 10.27 & 0.38 & 80.78 & 0.16 \\
\hline 2 & $27-06-200108: 07$ & $29-06-200100: 17$ & 40.17 & 78.43 & 30.13 & 31.43 & 1.49 & 0.25 & 2.30 & 35.73 & 9.57 & 3.73 & 5.00 & 0.33 & 0.50 & 9.98 & 8.47 & 19.95 & 0.66 \\
\hline 3 & $17-07-200105: 32$ & $21-07-2001 \quad 16: 52$ & 107.33 & 135.80 & 92.39 & 41.88 & 2.63 & 0.29 & 16.53 & 77.87 & 34.82 & 2.24 & 3.00 & 0.42 & 0.50 & 9.47 & 1.53 & 53.72 & 0.23 \\
\hline 4 & 02-08-2001 15:27 & $10-08-200122: 47$ & 199.33 & 63.30 & 44.03 & 18.95 & 1.24 & 0.27 & 27.90 & 86.38 & 55.80 & 1.55 & 13.00 & 0.25 & 1.00 & 14.29 & 4.27 & 85.08 & 0.61 \\
\hline 5 & 09-06-2002 13:52 & $16-06-200211: 52$ & 166.00 & 61.27 & 54.84 & 27.10 & 1.43 & 0.37 & 35.73 & 75.58 & 55.63 & 1.36 & 13.00 & 0.22 & 1.08 & 23.67 & 18.73 & 79.84 & 0.75 \\
\hline 6 & $19-08-2002$ 05:32 & $22-08-200204: 37$ & 71.08 & 113.97 & 43.20 & 37.53 & 3.95 & 0.37 & 27.28 & 102.48 & 53.13 & 1.93 & 8.00 & 0.44 & 1.00 & 9.37 & 3.77 & 16.81 & 0.68 \\
\hline 7 & 05-10-2002 06:12 & 08-10-2002 00:07 & 65.92 & 49.43 & 31.12 & 25.62 & 2.77 & 0.39 & 10.59 & 69.32 & 36.20 & 1.92 & 9.00 & 0.47 & 1.83 & 13.83 & 10.35 & 143.45 & 0.76 \\
\hline 8 & 02-06-2003 11:22 & 05-06-2003 17:37 & 78.25 & 67.23 & 28.91 & 24.53 & 1.39 & 0.19 & 1.83 & 36.31 & 14.44 & 2.52 & 12.00 & 0.37 & 1.33 & 9.77 & 4.92 & 30.33 & 0.76 \\
\hline 9 & $17-06-2003 \quad 17: 17$ & $20-06-2003$ 00:02 & 54.75 & 59.17 & 37.69 & 21.68 & 1.32 & 0.22 & 3.08 & 32.84 & 12.24 & 2.68 & 7.00 & 0.18 & 0.33 & 8.65 & 3.08 & 106.13 & 0.78 \\
\hline 10 & $30-06-200307: 57$ & $05-07-2003 \quad 17: 37$ & 129.67 & 81.73 & 68.56 & 21.88 & 1.52 & 0.27 & 15.39 & 70.14 & 35.70 & 1.96 & 14.00 & 0.15 & 0.42 & 14.21 & 5.35 & 53.77 & 0.27 \\
\hline 11 & 20-07-2004 04:12 & $23-07-2004$ 08:07 & 75.92 & 52.97 & 39.52 & 21.68 & 1.17 & 0.34 & 11.06 & 42.03 & 21.81 & 1.93 & 15.00 & 0.29 & 0.83 & 11.87 & 4.52 & 9.25 & 0.86 \\
\hline 12 & $10-09-200423: 47$ & 12-09-2004 04:17 & 28.50 & 61.60 & 16.10 & 22.43 & 2.33 & 0.30 & 10.83 & 55.83 & 26.89 & 2.08 & 17.00 & 0.45 & 1.08 & 14.02 & 4.22 & 67.10 & 0.55 \\
\hline 13 & 29-04-2005 01:32 & $11-05-200513: 47$ & 300.25 & 59.27 & 50.94 & 18.26 & 1.37 & 0.32 & 47.22 & 116.53 & 68.59 & 1.70 & 8.00 & 0.17 & 0.50 & 13.06 & 4.96 & 63.02 & 0.33 \\
\hline 14 & $29-05-2005$ 21:22 & 05 23:02 & 49.67 & 34.13 & 20.89 & 17.18 & 1.33 & 0.48 & 13.30 & 33.85 & 20.82 & 1.63 & 12.00 & 0.26 & 0.83 & 15.23 & 5.02 & 52.76 & 0.84 \\
\hline 15 & 03-06-2005 15:12 & 06-06-2005 11:42 & 68.50 & 45.80 & 33.98 & 14.14 & 1.03 & 0.29 & 7.18 & 40.39 & 22.10 & 1.83 & 10.00 & 0.25 & 0.58 & 13.28 & 5.21 & 87.85 & 0.50 \\
\hline 16 & $29-06-200500: 27$ & 03-07-2005 00:27 & 96.00 & 92.33 & 33.47 & 41.19 & 4.43 & 0.36 & 37.68 & 138.45 & 78.02 & 1.77 & 11.00 & 0.36 & 1.17 & 6.46 & 4.91 & 38.70 & 1.58 \\
\hline 17 & $18-07-2005 \quad 22: 42$ & $00521: 52$ & 311.17 & 130.77 & 98.47 & 36.37 & 2.50 & 0.28 & 44.18 & 124.88 & 70.28 & 1.78 & 10.00 & 0.28 & 0.83 & 13.39 & 7.61 & 62.03 & 0.52 \\
\hline 18 & $24-08-200608: 17$ & $31-08-200622: 47$ & 182.50 & 56.20 & 35.49 & 22.01 & 1.49 & 0.25 & 23.08 & 80.91 & 51.42 & 1.57 & 27.00 & 0.29 & 0.92 & 11.74 & 5.73 & 103.27 & 0.74 \\
\hline 19 & $29-09-2006$ 16:02 & $07-10-200621: 17$ & 197.25 & 39.40 & 33.19 & 13.34 & 1.54 & 0.32 & 29.23 & 70.16 & 45.43 & 1.54 & 6.00 & 0.50 & 1.25 & 13.59 & 4.53 & 82.81 & 0.59 \\
\hline 20 & $08-11-2006$ 17:02 & $15-11-200610: 52$ & 161.83 & 19.77 & 18.51 & 10.63 & 1.12 & 0.42 & 24.52 & 88.23 & 55.54 & 1.59 & 15.00 & 0.24 & 0.67 & 19.00 & 12.34 & 124.63 & 0.78 \\
\hline 21 & $24-05-2007$ 22:07 & $29-05-2007$ 19:02 & 116.92 & 88.50 & 85.05 & 25.80 & 1.58 & 0.51 & 26.97 & 60.99 & 39.05 & 1.56 & 4.00 & 0.27 & 0.75 & 15.22 & 4.51 & 32.53 & 0.58 \\
\hline 22 & $06-08-2007$ 14:17 & $10-08-200720: 02$ & 101.75 & 182.43 & 100.01 & 79.33 & 4.37 & 0.59 & 46.83 & 184.46 & 80.74 & 2.28 & 1.00 & 1.50 & 1.50 & 7.05 & 2.34 & 46.28 & 0.32 \\
\hline 23 & $18-08-200716: 22$ & $23-08-200722: 02$ & 125.67 & 94.57 & 47.69 & 26.57 & 3.32 & 0.39 & 43.68 & 112.10 & 74.62 & 1.50 & 6.00 & 0.15 & 0.25 & 9.67 & 5.91 & 330.95 & 1.49 \\
\hline 24 & $14-05-2008$ 12:02 & $18-05-200801: 47$ & 85.75 & 135.27 & 127.91 & 67.80 & 3.47 & 0.29 & 5.93 & 91.52 & 19.12 & 4.79 & 4.00 & 0.31 & 0.58 & 6.95 & 5.48 & 77.29 & 0.83 \\
\hline 25 & $22-01-2009$ 12:47 & $24-01-2009$ 14:42 & 49.92 & 27.47 & 19.47 & 14.29 & 1.80 & 0.58 & 17.71 & 46.73 & 31.76 & 1.47 & 11.00 & 0.42 & 1.08 & 17.32 & 9.36 & 56.01 & 0.82 \\
\hline 26 & $16-07-2009$ 15:22 & 09 18:12 & 218.83 & 4.23 & 84.16 & 31.49 & 1.66 & 0.2 & 36.08 & 73.13 & 52.89 & 138 & 7.00 & 0.29 & 0.67 & 14.51 & 6.71 & 76.76 & 0.49 \\
\hline 27 & 06-08-2009 20:47 & $13-08-2009$ 16:12 & 163.42 & 27.63 & 29.76 & 14.50 & 1.38 & 0.26 & 17.81 & 46.39 & 29.35 & 1.5 & 22.00 & 0.24 & 1.08 & 19.89 & 13.09 & 104.99 & 0.86 \\
\hline 28 & 25-07-2010 06:07 & $30-07-201002: 37$ & 116.50 & 75.47 & 45.94 & 20.70 & 1.74 & 0.34 & 31.39 & 75.31 & 49.40 & 1.52 & 4.00 & 0.42 & 0.92 & 8.82 & 2.64 & 83.60 & 0.42 \\
\hline 29 & $10-08-2010$ 15:47 & $12-08-201004: 37$ & 36.83 & 72.57 & 49.90 & 43.49 & 2.46 & 0.45 & 10.74 & 59.63 & 20.81 & 2.87 & 6.00 & 0.49 & 1.83 & 9.15 & 7.06 & 44.24 & 0.75 \\
\hline 30 & $25-08-2010 \quad 15: 32$ & 01-09-2010 02:27 & 154.92 & 60.27 & 42.10 & 18.72 & 2.19 & 0.44 & 47.47 & 120.27 & 78.13 & 1.54 & 16.00 & 0.31 & 1.67 & 12.34 & 5.72 & 112.53 & 0.63 \\
\hline 31 & $17-01-2011$ 14:57 & $20-01-2011 \quad 10: 42$ & 6775 & 2943 & 2969 & 16.28 & 084 & 031 & 6.23 & 28.79 & 16.91 & 17 & 7.00 & 0.42 & 1.50 & 10.00 & 3.92 & 88.99 & 0.66 \\
\hline 32 & 04-06-2011 18:42 & 08-06-2011 11:12 & 88.50 & 96.70 & 44.82 & 41.74 & 2.41 & 0.32 & 13.66 & 89.42 & 41.10 & 2.18 & 15.00 & 0.27 & 1.08 & 7.83 & 4.59 & 25.72 & 1.05 \\
\hline 33 & $11-08-201103: 02$ & $16-08-2011 \quad 13: 47$ & 130.75 & 55.07 & 36.90 & 17.47 & 1.40 & 0.29 & 20.90 & 53.62 & 38.00 & 1.41 & 5.00 & 0.35 & 0.67 & 12.31 & 4.86 & 69.10 & 0.67 \\
\hline 34 & $23-08-2012$ 20:22 & $26-08-2012$ 22:12 & 73.83 & 53.77 & 37.89 & 19.19 & 1.75 & 0.40 & 14.79 & 56.55 & 29.16 & 1.94 & 11.00 & 0.20 & 0.58 & 13.91 & 6.79 & 99.17 & 0.67 \\
\hline 35 & 07-05-2013 09:12 & 09-05-2013 12:52 & 51.67 & 67.93 & 62.69 & 32.58 & 1.50 & 0.29 & 0.88 & 39.08 & 9.22 & 4.2 & 4.00 & 0.60 & 1.00 & 7.75 & 4.38 & 295.46 & 0.85 \\
\hline 36 & 19-06-2013 18:02 & $25-06-2013 \quad 15: 12$ & 141.17 & 364.70 & 202.71 & 110.81 & 5.40 & 0.29 & 16.73 & 155.09 & 58.70 & 2.64 & 3.00 & 0.53 & 0.67 & 11.25 & 4.04 & 357.37 & 0.41 \\
\hline 37 & $22-07-2013 \quad 11: 42$ & $31-07-2013$ 07:02 & 211.33 & 121.23 & 95.53 & 59.63 & 3.16 & 0.22 & 13.41 & 125.56 & 39.62 & 3.17 & 14.00 & 0.36 & 0.92 & 7.30 & 6.85 & 119.31 & 1.61 \\
\hline 38 & $24-08-2013$ 13:32 & $26-08-2013 \quad 15: 27$ & 49.92 & 56.53 & 56.00 & 19.95 & 0.93 & 0.26 & 2.33 & 24.46 & 9.75 & 2.51 & 3.00 & 0.36 & 0.92 & 9.32 & 3.38 & 245.25 & 0.61 \\
\hline 39 & $06-09-2013$ 13:57 & $14-09-2013$ 18:57 & 197.00 & 93.87 & 55.13 & 34.85 & 2.18 & 0.37 & 61.10 & 110.71 & 86.76 & 1.28 & 11.00 & 0.24 & 0.58 & 12.96 & 6.09 & 340.25 & 1.80 \\
\hline
\end{tabular}


Table C: Sampled 24-hour extreme events

\begin{tabular}{|c|c|c|c|c|c|c|c|c|c|c|c|c|c|c|c|c|c|c|c|}
\hline $\begin{array}{l}24- \\
\text { hour }\end{array}$ & start & end & $\begin{array}{l}\text { duration } \\
{[\mathrm{h}]}\end{array}$ & $\begin{array}{l}\max \\
15 \mathrm{~min} \\
{[\mathrm{~mm} / \mathrm{hr}]}\end{array}$ & $\begin{array}{l}\text { ratio } \\
15 \text { min } \\
{[-]}\end{array}$ & $\begin{array}{l}\max \\
1 \mathrm{hr} \\
{[\mathrm{mm} / \mathrm{hr}]}\end{array}$ & $\begin{array}{l}\max \\
24 \mathrm{hr} \\
{[\mathrm{mm} / \mathrm{hr}]}\end{array}$ & $\begin{array}{l}\text { mean } \\
\text { wet A } \\
{[-]}\end{array}$ & $\begin{array}{l}\min \\
\text { depth } \\
{[\mathrm{mm}]}\end{array}$ & $\begin{array}{l}\text { max } \\
\text { depth } \\
{[\mathrm{mm}]}\end{array}$ & $\begin{array}{l}\text { mean } \\
\text { depth } \\
{[\mathrm{mm}]}\end{array}$ & $\begin{array}{l}\text { ratio } \\
\text { depth } \\
{[-]}\end{array}$ & $\begin{array}{l}\text { cell } \\
\text { num } \\
{[-]}\end{array}$ & $\begin{array}{l}\text { cell life } \\
\text { mean } \\
{[\mathrm{h}]}\end{array}$ & $\begin{array}{l}\text { cell life } \\
\text { max } \\
\text { [h] }\end{array}$ & $\begin{array}{l}\text { mean } \\
\text { vel } \\
{[\mathrm{m} / \mathbf{s}]}\end{array}$ & $\begin{array}{l}\text { sd } \\
\text { vel } \\
{[-]}\end{array}$ & $\begin{array}{l}\text { mean } \\
\text { dir } \\
\text { [deg.] }\end{array}$ & $\begin{array}{l}\text { sd } \\
\text { dir } \\
{[-]}\end{array}$ \\
\hline 1 & 01-01-2001 03:27 & 11-01-2001 09:02 & 245.58 & 27.70 & 29.11 & 10.89 & 2.48 & 0.47 & 50.17 & 101.68 & 71.75 & 1.42 & 3.00 & 0.33 & 0.58 & 17.65 & 4.46 & 77.43 & 0.24 \\
\hline 2 & $17-07-200105: 32$ & $21-07-2001 \quad 16: 52$ & 107.33 & 135.80 & 92.39 & 41.88 & 2.63 & 0.29 & 16.53 & 77.87 & 34.82 & 2.24 & 3.00 & 0.42 & 0.50 & 9.47 & 1.53 & 53.72 & 0.23 \\
\hline 3 & 30-08-2001 09:02 & $17-09-200121: 12$ & 444.17 & 58.70 & 49.62 & 20.81 & 2.33 & 0.34 & 89.37 & 204.38 & 153.62 & 1.33 & 10.00 & 0.28 & 0.92 & 9.71 & 5.27 & 75.33 & 0.61 \\
\hline 4 & 19-09-2001 08:02 & 23-09-2001 14:37 & 102.58 & 68.97 & 39.02 & 22.49 & 3.12 & 0.34 & 24.19 & 101.56 & 51.77 & 1.96 & 5.00 & 0.18 & 0.33 & 11.28 & 3.51 & 119.75 & 0.43 \\
\hline 5 & 06-11-2001 02:17 & 09-11-2001 03:17 & 73.00 & 29.57 & 20.83 & 9.30 & 2.19 & 0.43 & 24.78 & 66.21 & 44.35 & 1.49 & 7.00 & 0.23 & 0.42 & 14.52 & 5.52 & 96.13 & 0.47 \\
\hline 6 & $19-08-2002 \quad 05: 32$ & $22-08-200204: 37$ & 71.08 & 113.97 & 43.20 & 37.53 & 3.95 & 0.37 & 27.28 & 102.48 & 53.13 & 1.93 & 8.00 & 0.44 & 1.00 & 9.37 & 3.77 & 16.81 & 0.68 \\
\hline 7 & 05-10-2002 06:12 & 08-10-2002 00:07 & 65.92 & 49.43 & 31.12 & 25.62 & 2.77 & 0.39 & 10.59 & 69.32 & 36.20 & 1.92 & 9.00 & 0.47 & 1.83 & 13.83 & 10.35 & 143.45 & 0.76 \\
\hline 8 & 07-11-2002 00:27 & 11-11-2002 16:12 & 111.75 & 46.10 & 26.29 & 15.78 & 2.28 & 0.54 & 39.27 & 100.08 & 69.23 & 1.45 & 3.00 & 0.08 & 0.17 & 7.13 & 5.42 & 135.00 & 0.66 \\
\hline 9 & $23-12-2002$ 22:47 & $31-12-200208: 57$ & 178.17 & 16.40 & 13.38 & 8.75 & 2.31 & 0.52 & 55.65 & 127.49 & 90.00 & 1.42 & 37.00 & 0.32 & 1.17 & 15.85 & 6.69 & 89.80 & 0.67 \\
\hline 10 & 03-10-2003 04:07 & $12-10-200303: 17$ & 215.17 & 28.10 & 18.14 & 14.36 & 2.64 & 0.44 & 62.73 & 150.40 & 99.83 & 1.51 & 25.00 & 0.33 & 1.08 & 14.25 & 7.52 & 97.94 & 0.59 \\
\hline 11 & $11-12-200310: 57$ & $16-12-200320: 47$ & 129.83 & 34.00 & 20.16 & 16.13 & 3.79 & 0.50 & 31.30 & 131.59 & 80.16 & 1.64 & 14.00 & 0.22 & 0.75 & 17.67 & 6.54 & 106.59 & 0.71 \\
\hline 12 & 19-01-2004 04:02 & 20-01-2004 06:22 & 26.33 & 10.77 & 6.63 & 6.32 & 2.26 & 0.89 & 21.70 & 54.18 & 34.50 & 1.57 & 41.00 & 0.36 & 3.08 & 15.37 & 11.90 & 157.76 & 1.27 \\
\hline 13 & 06-05-2004 09:52 & $406: 02$ & 44.17 & 10.33 & 7.83 & 7.78 & 2.44 & 0.83 & 32.20 & 63.58 & 46.57 & 1.37 & 51.00 & 0.48 & 5.25 & 9.18 & 8.83 & 228.73 & 1.13 \\
\hline 14 & $10-09-200423: 47$ & $12-09$ & 28.50 & 61.60 & 16.10 & 22.43 & 2 & 0.30 & 10.83 & 55.83 & 26.89 & 2.08 & 17.00 & 0.45 & 1.08 & 14.02 & 4.22 & 67.10 & 0.55 \\
\hline 15 & 20-09-2004 07:32 & $30-09-200420: 07$ & 252.58 & 27.97 & 24.79 & 12.08 & 0 & 0.35 & 51.86 & 96.32 & 79.12 & 1.22 & 20.00 & 0.21 & 0.58 & 14.72 & 6.18 & 121.42 & 0.54 \\
\hline 16 & $15-11-2004$ 17:02 & 24-11-2004 04:47 & 203.75 & 17.00 & 14.06 & 11.93 & 3.69 & 0.51 & 59.93 & 154.06 & 104.03 & 1.48 & 38.00 & 0.21 & 0.67 & 14.77 & 6.56 & 130.15 & 0.75 \\
\hline 17 & 5 19:07 & $26-0$ & 199.83 & 32.30 & 24.95 & 5 & 6 & 0.46 & 53.55 & 127.98 & 95.98 & 1.33 & 18.00 & 35 & 2.08 & 12.48 & 7.84 & 127.90 & 1.06 \\
\hline 18 & 09-02-2005 18:02 & 05 18:27 & 168.42 & 30.90 & 26.15 & 10.62 & 2.8 & 0.56 & 48.87 & 153.18 & 103.46 & 1.48 & 4.00 & 0.15 & 0.17 & 23.58 & 5.40 & 103.37 & 0.15 \\
\hline 19 & $29-06-200500: 27$ & 03-07-2005 00:27 & 96.00 & 92.33 & 33.47 & 41.19 & 4.43 & 0.36 & 37.68 & 138.45 & 78.02 & 1.77 & 11.00 & 0.36 & 1.17 & 6.46 & 4.91 & 38.70 & 1.58 \\
\hline 20 & $24-03-2006$ 10:32 & 05-04-2006 19:27 & 296.92 & 32.73 & 31.51 & 11.73 & 1.72 & 0.43 & 48.83 & 132.16 & 89.28 & 1.48 & 6.00 & 0.29 & 0.50 & 17.67 & 7.41 & 97.58 & 0.55 \\
\hline 21 & $15-05-200618: 37$ & $605: 12$ & 58 & 43.23 & 30.09 & 4 & 2. & 0.47 & 78.55 & 186.32 & 143.86 & 1.30 & 10.00 & 0.19 & 0.42 & 16.83 & 7.51 & 74.33 & 0.38 \\
\hline 22 & 29-09-2006 16:02 & $07-10-200621: 17$ & 197.25 & 39.40 & 33.19 & 13.34 & 1.54 & 0.32 & 29.23 & 70.16 & 45.43 & 1.54 & 6.00 & 0.50 & 1.25 & 13.59 & 4.53 & 82.81 & 0.59 \\
\hline 23 & $10-12-200623: 07$ & $13-12-2006 \quad 15: 37$ & 64.50 & 9.43 & 6.84 & 7.80 & 1.75 & 0.64 & 14.94 & 48.64 & 35.61 & 1.37 & 52.00 & 0.43 & 4.33 & 13.27 & 9.51 & 106.52 & 1.14 \\
\hline 24 & 06-05-2007 17:42 & $17-05-2007$ 15:57 & 262.25 & 79.07 & 69.61 & 20.81 & 2.45 & 0.53 & 60.10 & 184.69 & 120.20 & 1.54 & 9.00 & 0.39 & 0.92 & 16.49 & 4.34 & 103.48 & 0.41 \\
\hline 25 & 06-08-2007 14:17 & $10-08-200720: 02$ & 101.75 & 182.43 & 100.01 & 79.33 & 4.37 & 0.59 & 46.83 & 184.46 & 80.74 & 2.28 & 1.00 & 1.50 & 1.50 & 7.05 & 2.34 & 46.28 & 0.32 \\
\hline 26 & $18-08-2007 \quad 16: 22$ & $23-08-$ & 67 & 94.57 & 47.69 & 7 & 3 & 0 & 43.68 & 112.10 & 74.62 & 1.50 & 6.00 & 15 & 0.25 & 9.67 & 5.91 & 330.95 & 1.49 \\
\hline 27 & 02-11-2007 01:37 & $704: 07$ & 50 & 22.77 & 21.20 & 0 & 19 & 0.40 & 54.03 & 132.81 & 101.55 & 1.31 & 19.00 & 0.30 & 0.83 & 17.27 & 7.40 & 146.91 & 0.85 \\
\hline 28 & 29-11-2007 05:52 & $11-12-2007$ 17:32 & 299.67 & 20.27 & 17.81 & 10.63 & 2.48 & 0.50 & 66.04 & 175.95 & 136.15 & 1.29 & 20.00 & 0.23 & 0.58 & 17.94 & 6.06 & 102.20 & 0.56 \\
\hline 29 & 29-09-2008 12:32 & 09-10-2008 23:42 & 251.17 & 30.60 & 26.39 & 11.46 & 2.30 & 0.47 & 53.53 & 143.60 & 100.53 & 1.43 & 14.00 & 0.34 & 1.33 & 18.32 & 8.85 & 92.24 & 0.67 \\
\hline 30 & $10-08-2010 \quad 15: 47$ & $12-08-201004: 37$ & 36.83 & 72.57 & 49.90 & 43.49 & 2.46 & 0.45 & 10.74 & 59.63 & 20.81 & 2.87 & 6.00 & 0.49 & 1.83 & 9.15 & 7.06 & 44.24 & 0.75 \\
\hline 31 & 02-11-2010 12:32 & $15-11$ & 31917 & 2407 & 23.05 & 11.98 & 27 & $0<-2$ & 9121 & 188.89 & 142.05 & 1.33 & 34.00 & 0.35 & 1.75 & 16.31 & 9.14 & 96.32 & 0.84 \\
\hline 32 & 11-01-2011 09:12 & $15-01-2011$ 19:22 & 106.17 & 21.47 & 18.06 & 9.53 & 2.74 & 0.67 & 33.32 & 109.95 & 73.09 & 1.50 & 5.00 & 0.45 & 0.92 & 16.04 & 6.42 & 96.76 & 0.71 \\
\hline 33 & 11-12-2011 17:52 & $18-12-201120: 22$ & 170.50 & 19.37 & 18.12 & 11.38 & 1.90 & 0.54 & 41.98 & 94.40 & 73.31 & 1.29 & 36.00 & 0.31 & 2.50 & 17.67 & 16.23 & 99.21 & 1.12 \\
\hline 34 & $18-01-2012 \quad 15: 22$ & $24-01-2012$ 12:02 & 140.67 & 19.00 & 15.92 & 7.03 & 2.03 & 0.51 & 47.59 & 99.88 & 73.09 & 1.37 & 24.00 & 0.24 & 1.17 & 20.18 & 9.10 & 123.92 & 0.80 \\
\hline 35 & 02-10-2012 06:47 & 07-10-2012 02:17 & & 27.47 & 16.86 & 15.17 & 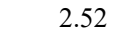 & 0.47 & 36.25 & 90.95 & 64.61 & 1.41 & 31.00 & 0.30 & 0.83 & 18.31 & 9.72 & 81.79 & 0.59 \\
\hline 36 & $20-12-2012 \quad 12: 57$ & 04-01-2013 09:37 & 356.67 & 26.13 & 25.63 & 12.73 & 2.97 & 0.51 & 75.56 & 178.28 & 133.77 & 1.33 & 27.00 & 0.34 & 1.50 & 15.25 & 6.70 & 93.74 & 0.72 \\
\hline 37 & 19-06-2013 18:02 & $25-06-2013$ 15:12 & 141.17 & 364.70 & 202.71 & 110.81 & 5.40 & 0.29 & 16.73 & 155.09 & 58.70 & 2.64 & 3.00 & 0.53 & 0.67 & 11.25 & 4.04 & 357.37 & 0.41 \\
\hline 38 & 22-07-2013 11:42 & $31-07-2013$ 07:02 & 211.33 & 121.23 & 95.53 & 59.63 & 3.16 & 0.22 & 13.41 & 125.56 & 39.62 & 3.17 & 14.00 & 0.36 & 0.92 & 7.30 & 6.85 & 119.31 & 1.61 \\
\hline 39 & 06-09-2013 13:57 & $14-09-2013$ 18:57 & 197.00 & 93.87 & 55.13 & 34.85 & 2.18 & 0.37 & 61.10 & 110.71 & 86.76 & 1.28 & 11.00 & 0.24 & 0.58 & 12.96 & 6.09 & 340.25 & 1.80 \\
\hline
\end{tabular}




\section{S2. Hierarchical Clustering method}

The hierarchical clustering methods selected are the Ward method and the Average Linkage Method (Murtagh, 1983). The methods are agglomerative clustering methods where all events start in separate clusters, the two less dissimilar events are joined until all events are in one cluster (Cormack, 1971). The dissimilarities between events are calculated based on the Lance-Williams cluster update method(Lance and Williams, 1966). The results are illustrated using dendrograms. Dendrograms for Ward and and Average linkage methods can be seen in Figure A, top and bottom respectively.
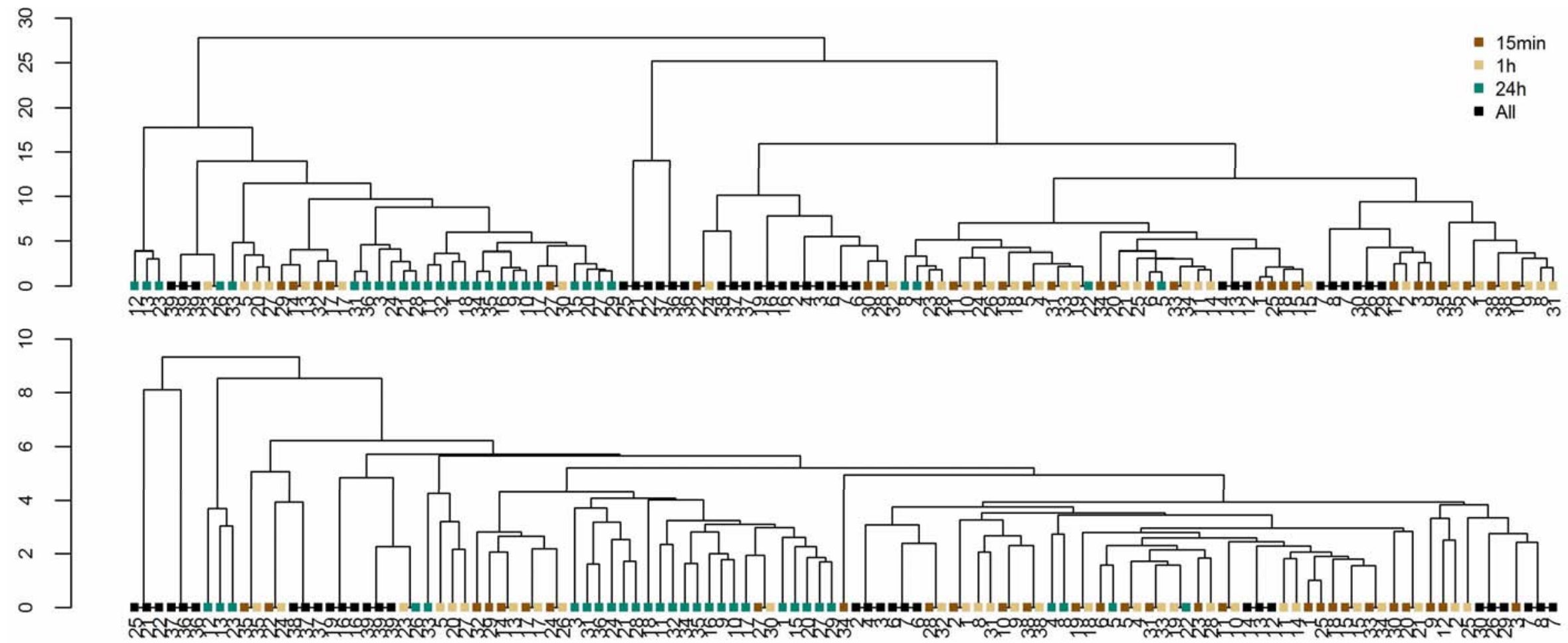

Figure A: Dendrogram performed on the combined dataset of 15-min, 1-hour and 24-hour extreme events with Ward (top) and Average linkage (bottom) method. 
S3. References

Cormack, R. M.: A Review of Classification, J. R. Stat. Soc. Ser. A, 134(3), 321-367, 1971.

Lance, G. N. and Williams, W. T.: A generalized sorting strategy for computer classifications, Nature, 212, 218, doi:10.1038/212218a0, 1966.

Murtagh, F.: A Survey of Recent Advances in Hierarchical Clustering Algorithms, Comput. J., 26(4), 354-359, doi:10.1093/comjnl/26.4.354, 1983. 